\title{
OSSERVAZIONI SOPRA LA LINGUA DELLE NOVELLE DI ARRIGO BOITO ${ }^{1}$
}

\begin{abstract}
L'articolo studia gli aspetti fonologici, morfologici, grafici, lessicali e sintattici delle cinque novelle di Arrigo Boito (L'alfier nero, Iberia, La musica in piazza, Il pugno chiuso e Il trapezio) composte nel breve periodo compreso tra il 1867 e il 1874 . Sono stati raccolti e contemplati, tra i fenomeni linguistici della prosa boitiana, i tratti maggiormente rilevanti che contribuiscono ad una complessiva cura euritmica del discorso, quali ad esempio il ricorrere di poetismi e di una terminologia spiccatamente desueta o tecnica, di dantismi, dialettalismi o forestierismi: mediante uno spoglio complessivo è stato rilevato il carattere sperimentale della scrittura di Boito e si è potuta comparare l'espressione individuale del letterato musicista con gli stilemi dell'Ottocento italiano (in particolare, della temperie scapigliata), eventualmente in rapporto agli altri generi, poetici e librettistici, cui lo stesso autore si dedicò.
\end{abstract}

Parole chiave: Arrigo Boito, novelle, lingua, Scapigliatura, Ottocento.

\section{INTRODUZIONE}

L'attenzione linguistica di Boito, la sua ricerca formale, è un tratto espressivo necessario, se non vincolante, della propria scrittura: tale sperimentalismo è da intendersi come la costante volontà di sintetizzare le forme più propriamente letterarie, se non arcaiche, con i più moderni prodotti linguistici, quest'ultimi attinenti in prevalenza alla sfera di un lessico tecnico, derivato dall'interesse dell'autore per la scienza, la musica, gli scacchi, la

*giulio.nardo1@gmail.com

${ }^{1}$ Le cinque novelle prese in esame sono: L'alfier nero [AN], Iberia [IB], La musica in piazza [MP], Il pugno chiuso [PC], Il trapezio [TR]. Il vaglio è stato effettuato limitatamente all'edizione integrale curata da Angela Ida Villa (2009) cui, salvo diversa indicazione, si farà riferimento. Si noti inoltre che, relativamente all'Alfier nero, l'analisi si è concentrata principalmente sulla seconda redazione della novella, apparsa nella "Strenna Italiana" nel 1868. Nel corso del lavoro, si è tenuto comunque in considerazione il testo della prima redazione, pubblicata nel "Politecnico" (Boito 1867), così da effettuare eventuali raffronti. 
cultura spagnola ed orientale. Lo stile si presenta ordinato, calibrato ed elegante, capace nel contempo di servirsi sia di una terminologia desueta, poetica, preziosa, sia di tecnicismi e di espressioni di attestazione recente nel panorama italiano ottocentesco.

\section{VOCALISMO}

Si riscontra un ampio utilizzo del dittongo -uo- dopo consonante palatale, sia in posizione atona che tonica. Si vedano le voci: giuocatore, giuoco, giuocare $^{2}$, spagnuolo, figliuolo, gragnuola, cagnuolo, usignuolo, ecc. In altre situazioni, si osserva come dall'Alfier nero al Trapezio l'impiego del dittongo risulti sempre più controbilanciato dalla monottongazione in - $\mathrm{O}^{-}$. Ai casi di muovere, esiti esclusivi nelle prime due novelle, fanno successivamente riscontro momenti di oscillazione tra muovere [PC: 232; TR: 245, 264] e movere [PC: 225, 235; TR: 239, 243]. Altri esempi coinvolgono i termini scuotere [TR: 246] e l'allotropo scotere [MP: 209], (ri)suonare [PC: 228; MP: 261, ecc.; TR: 251] e (ri)sonare [IB: 183, 191; MP: 210; TR: 257]. Risulta esclusivo foco [MP: 205], confermato, a distanza di alcuni anni, seppure in posizione atona, da infocavano [TR: 251]. L'alternanza tra una forma dittongata o meno non risponde a regole prestabilite: nel complesso, Boito opta per l'esito maggiormente eufonico, talvolta per il meno comune. Si tenga presente che le cinque novelle sono caratterizzate da cure formali sostanzialmente diverse l'una dall'altra: ad una letterarietà evidente ma sempre contenuta nell' Alfier nero e nella Musica in piazza ${ }^{3}$, e notevolmente ridotta nel Pugno chiuso ${ }^{4}$, fa riscontro un'attenzione euritmica e lessicale

${ }^{2} \mathrm{Si}$ noti in particolare la correzione di giuocò [AN: 179] nella variante dittongata rispetto ad una precedente monottongata presente nel "Politecnico" o, al contrario, nella scelta opposta di monottongazione del verbo nella frase «non giocava ad un giuoco» [AN: 172] che attesta, rispetto al precedente giuocava (Nardi 1942: 404), un'attenzione eufonica propria della scrittura boitiana, qui volta ad evitare la ridondanza del suono -uo-, e presente anche nel libretto dell'Amleto: «si potrebbe / Giocar con esso al giuoco del palèo» (Nardi 1942: 537). Nelle sue novelle, Boito si serve esclusivamente delle forme dittongate giuocatore, giuoco, giuocare; uniche due eccezioni, ambedue in posizione atona, risultano appunto l'uscita monottongata in «non giocava ad un giuoco» ed in giocare [TR: 250], nel Trapezio. Nel Trapezio, però, a un'occorrenza atona monottongata fa riscontro anche una atona dittongata, in giuocarle [TR: 264].

${ }^{3}$ Nella quale, però, è preponderante una cifra stilistica che, più che al genere novellistico, accosta la prosa al genere delle Cronache dei concerti e dei teatri, e quindi al taglio giornalistico (Nardi 1942: 1067-1223).

${ }^{4}$ Ridotta, ma a volte attraversata da esiti quali le monottongazioni di commovere [PC: 230] e percotere [PC: 237, 251], quest'ultimo oscillante con un'attestazione dell'allotropo percuotere [PC: 224-225]. 
altamente ponderata in Iberia e nel Trapezio. Se Manzoni, nella Quarantana, aveva provveduto a normalizzare gli esiti dittongati nei corrispettivi monottongati in $-\mathrm{O}^{-}$, ancora per tutto il secondo Ottocento si sarebbero attestate oscillazioni tra gli allotropi, spesso generate dalla persistenza di residui letterari della tradizione, oppure dalla volontà di mantenimento, da parte di certi autori, di cifre consapevoli atte ad affermare la preziosità nella produzione prosastica ${ }^{6}$. Nel caso di Boito, le alternanze tra un esito e l'altro sono rilevabili anche nel corso della sua produzione librettistica e poetica ${ }^{7}$. Tra le altre forme di dittongamento, si rilevano i casi di intiera [AN: 177, TR: 269$]^{8}$ contro quelli di intero/-a [PC: 224, 227] ${ }^{9}$. La propensione di Boito per la sonorità più letteraria del dittongo tonico è evidente negli usi, ancora comuni nell'Ottocento ${ }^{10}$, di altiera [MP: 205] e leggiero/-a [IB: 182, TR: 257, 263, 270]. Per i dittonghi atoni, si segnalano le forme marcate di tiepore [TR: 263] ${ }^{11}$ e dieciotto [IB: due occorrenze in 182; TR: 255] ${ }^{12}$. Al contrario, un mancato dittongamento con conseguente esito letterario è riscontrabile nel participio passato quetato [TR: 270$]^{13}$.

A proposito del vocalismo sono stati riscontrati numerosi casi di scelte stilistiche che confermerebbero un'attenzione conservativa del materiale linguistico trattato. Tra i vari, si citano: gli atoni dilicato [IB: 185; MP: 200;

${ }^{5}$ Cfr. Marazzini (1994: 406). Unica eccezione, figliuolo (cfr. Colombo 2011: 47).

${ }^{6}$ Per esempio, Fogazzaro (cfr. Colombo 2011: 94; Migliorini 1960: 679).

${ }^{7}$ In posizione accentata, giuoco è rintracciabile nel Mefistofele (Nardi 1942: 122) che oscilla, nello stesso libretto, con l'allotropo gioco (Nardi 1942: 106), attestato anche in Dualismo, nel Libro dei versi (Villa 2009: 53). Si noti peraltro che, se nella prosa e in Dualismo (Ibid.) Boito seleziona l'esclusivo foco, ormai marcatamente poetico per il tempo (cfr. TB), nel libretto di Otello ricorre alla forma più comune di fuoco, attestato in due momenti (Nardi 1942: 880, 884).

${ }^{8} \mathrm{Ma}$ intiero/-a godeva ancora «di ampia diffusione nella lingua ottocentesca» (cfr. Koban 2017: 86).

${ }^{9}$ Ai quali si aggiungono i casi esclusivi e meno marcati di intero/-a tratti da Iberia [IB: 181, 184, 185, 198], interessanti da notare in quanto situati all'interno di una novella caratterizzata da una forte cifra letteraria.

${ }^{10}$ Cfr. BI; GDLI; TB.

${ }^{11}$ Già considerato dal TB meno usato rispetto al monottongato tepore, cui rinvia. La BI non riporta alcun esempio.

${ }^{12}$ L'allotropo diciotto non è contemplato nella novellistica boitiana. Dieciotto è registrato nel TB, ma già per il tempo veniva considerato desueto (cfr. GDLI). La BI registra tre utilizzi esclusivamente nelle prose di Da Ponte e Leopardi; quindi, relegabili al primo Ottocento.

${ }^{13} \mathrm{Cfr}$. TB. 
TR: 270] ${ }^{14}$, ambidue [IB: 190, 191 $]^{15}$, gherofano $[\mathrm{IB}: 187]^{16}$, ricirculare [TR: 253,258$]^{17}$, o il desinenziale cavaliero [IB: 181, quattro casi in 182] ${ }^{18}$. $\mathrm{Al}$ contrario che nei libretti ${ }^{19}$, nella prosa è esclusivo il più comune gettare [MP: 109; TR: 258, 259].

La $i$ prostetica davanti a $s$ complicata ${ }^{20}$ si trova attuata frequentemente in Boito (ad esempio, «stavano per iscadere» [PC: 232], «non isbirciassero» [MP: 215], «non isgomentarla» [TR: 269]).

A proposito della vocale $i$, alcuni interessi grafici si presentano nell'introduzione della palatale $j^{21}$ dalla seconda redazione dell'Alfier nero (scrittojo [AN: 170], annojarsi [AN: 173], projettile [Ibid.], ecc.), il cui uso è riconfermato in Iberia. Nelle successive novelle, questa prima assidua attenzione per il grafema verrà meno ed il segno sarà normalizzato in $i$ (scrittoio [PC: 230]). Le rare eccezioni (joniche [MP: 218], solajo [PC: 227], granajo [TR: 242], ecc.) sembrano riconducibili a un retaggio passivo della tradizione. Un altro aspetto concerne l'uso degli accenti: in alcuni momenti, si leggono pestio [IB: 187], guaina [IB: 189], ballonzolio [MP: 209], mandòla [MP: 212], lavorio [TR: 248], lugùbre [TR: 262], rètina [TR: 266], ecc., a volte per indicare una lettura più etimologicamente corretta (guaina), in altre (lugùbre) per imporre attraverso la diastole una variante poetica $^{22}$. Interessante il caso della dieresi posta sopra coleöptero [MP: 202], atta a marcare la musicalità della parola.

${ }^{14}$ Nella riscrittura dell'Alfier nero si legge un delicata [AN: 170], che corregge il dilicata presente nella precedente redazione. Nel Trapezio, il dilicata segnalato oscilla con un caso a favore dell'esito in -e- [TR: 245].

${ }^{15}$ Desueto già per il TB.

${ }^{16}$ Forma antica e letteraria (cfr. GDLI), desueto (cfr. TB), il suo utilizzo rimane anteriore al sedicesimo secolo (cfr. BI). Nella Musica in piazza si legge garofano [MP: 215]. La scelta arcaizzante è senz'altro dettata dalla letterarietà insita in Iberia.

${ }^{17}$ Desueto già per il TB.

${ }^{18}$ Cui fanno riscontro, sempre in Iberia, alcune uscite in - $e$ [IB: due casi in 181, 182]. Essendo ancora attestato in Prati e Nievo (cfr. GDLI), cavaliero può essere inteso come variatio d'autore, sebbene nel secondo Ottocento la forma già si "stava ormai specializzando come poetica" (Buroni 2013: 144).

${ }^{19}$ Gittare, preceduto da croce nel TB, è recuperato nell'Otello: «Gittate i palischermi!» (Nardi 1942: 881).

${ }^{20}$ Il fenomeno, sebbene sempre più circoscritto, sopravvive per tutto il XIX secolo (cfr. Koban 2017: 99).

${ }^{21} \mathrm{Nel}$ secondo Ottocento, tale grafia appare ormai in forte regresso (cfr. Migliorini 1960: 699). Il suo uso si presenta ancora in Nievo (cfr. Motta 2015: 137; Zangrandi 2008: 31).

22 Attestata anche nella Gioconda: «La vidi tre volte scagliar su' tuoi remi / Parole tremende, - lugùbri anatèmi» (Nardi 1942: 625). 


\section{APOCOPE ED AFERESI}

L'apocope postconsonantica è un tratto sempre più in calo nel corso del XIX secolo ${ }^{23}$, inteso ora come carattere passivo della tradizione, ora come volontà di mantenimento di una cifra letteraria ${ }^{24}$. In Boito, il ricorso alle apocopi può consistere nell'intenzione di organizzare i suoni del discorso in una linea eufonica, ritmica, la quale, attraverso la ricerca di corrispondenze foniche o, appunto, di espedienti consolidati nella tradizione, affermerebbe il carattere letterario della sua scrittura. Tra le varie, si segnalano apocopi postconsonantiche in tempi verbali finiti («convien por mente alla professione» [TR: 252]) e infiniti («s'anco mi sia tolto il conversar delle labbra» [IB: 239]), nelle congiunzioni («eppur l'uno pareva composto coll'armonia dell'altro» [TR: 257]) ${ }^{25}$, negli avverbi («or seguendo coll'occhio» [TR: $239])^{26}$ e nelle preposizioni («fuor dalla gabbia!» [MP: 200]) $)^{27}$, ed apocopi postvocaliche coinvolgenti le preposizioni («il lungo studio de' scacchi» [AN: 174$]^{28}$, «co' suoi tortuosi meandri» [IB: 186], «materia pe' miei studi» $\left.[\mathrm{PC}: 224]^{29}\right)^{30}$.

${ }^{23}$ Cfr. Koban (2017: 100).

${ }^{24}$ Si vedano le prose di Carducci e D’Annunzio. Cfr. Serianni (2013: 197-199). Per i “troncamenti eufonici” di D’Annunzio, cfr. Coletti (1993: 310).

${ }^{25}$ Eppur è occorrenza esclusiva in Iberia [IB: 189, 195].

${ }^{26} \mathrm{Nel}$ Trapezio ed in Iberia, novelle dal carattere letterario, si contano rispettivamente undici ed una occorrenze di or, mentre l'uscita piana in ora risulta esclusiva nell'Alfier nero, nella Musica in piazza e nel Pugno chiuso.

${ }^{27}$ Interessante l'uso della preposizione fino nel Trapezio, dove l'apocope contribuisce ad esiti comuni («Fin da quando» [TR: 240], «fin dalla tenerissima infanzia» [TR: 245]), mentre, al contrario, l'unico caso di uscita piana, «fino dagli anni più teneri» [TR: 240], conferisce un sapore letterario all'espressione.

${ }^{28}$ Dove corregge un precedente degli della prima redazione.

${ }^{29}$ Si noti come l'unica occorrenza di pe' plurale apocopato si attesti nel Pugno chiuso, la novella dai toni meno letterari.

${ }^{30} \mathrm{Nel}$ complesso, le preposizioni apocopate accentuano il carattere poetico nella prosa boitiana. Confrontando le occorrenze del fenomeno nel corso delle cinque novelle, si evince come la sua prassi divenga sempre più consolidata nella scrittura. Inizialmente si tratta di un'applicazione consapevole, come attestano le correzioni di Boito apportate nella seconda redazione dell'Alfier nero. Nelle novelle successive, invece, il numero di apocopi accresce notevolmente, in particolare nel Trapezio, a séguito di uno stile sempre più complesso e letterario, poetico e conservativo. In questo caso, probabilmente si tratta di una strategia inconscia dell'autore, rafforzata di novella in novella e manifestatasi compiutamente nell'ultima, Il trapezio, la cui ricercatezza avrebbe indotto il prosatore a servirsi di tutti quegli stilemi che potevano contribuire al dettato poetico dell'espressione. 
Il fenomeno dell'aferesi, già in calo nel corso del XIX secolo, pur attestandosi raramente nella prosa boitiana, ammette comunque alcuni casi (lodola [TR: 256] ${ }^{31}$, briaco [IB: 198] ${ }^{32}$, sclamare $^{33}$ ).

\section{CONSONANTISMO}

Anche i casi di oscillazione delle occorrenze tra gli esiti in consonante sorda ed in sonora confermano quella ricerca boitiana volta a garantire le esigenze eufoniche così necessarie nella sua produzione: sebbene le forme selezionate dall'autore rientrino nella casistica dei fenomeni ancora largamente in uso, per quanto grandemente in calo nel corso dell'Ottocento, la loro presenza contribuisce a rafforzare la preziosità del tono linguistico delle novelle (per esempio, si vedano gli esclusivi lagrima in velare occlusiva sonora $^{34}$, brage $^{35}$, spaldo ${ }^{36}$, scuriada ${ }^{37}$ ).

Tra i nessi consonantici, sono rilevabili una palatalizzazione del nesso -ng- $(\text { ugne [TR: 264] })^{38} \mathrm{e}$ un esito in laterale palatale per capegli [IB: 191] ${ }^{39}$.

${ }^{31}$ Variante toscana (cfr. TB; GDLI; Fanfani 1976). Ma è anche milanesismo contemplato in Arrighi (1896) ed in Angiolini (1897).

${ }^{32}$ Ricomparirà anche nell'Otello (Nardi 1942: 245).

${ }^{33}$ Frequente ancora nell'Ottocento, ad esempio in Nievo (cfr. Motta 2015: 140). In Boito, la scelta tra l'esito aferetico e quello intero sembrerebbe rispondere ad esigenze eufoniche: sclamare si attesta dopo la battuta («"Il mio fiorino rosso!” sclamò l'avaro» [PC: 228], «"La mia!” sclamai» [TR: 261), mentre esclamare è adottato a introduzione della battuta («Il medico meravigliato, esclamò» [PC: 231], «drizzando il gesto alla statua esclamai: "La Gloria!"» [TR: 251]). Unica eccezione, prima della battuta, ma con esito aferetico: «mi guardò meravigliato e sclamò» [PC: 225], dove l'aferesi evita la ridondanza del suono $e$ tra la congiunzione ed il verbo.

${ }^{34}$ Nell'Ottocento, lagrima risulta più comune che il suo allotropo in sorda, lacrima (cfr. TB; GDLI; BI).

${ }^{35}$ Attestato in Dante, appare come variante arcaica di brace ancora in Monti, Fogazzaro e Pascoli (cfr. GDLI).

${ }^{36}$ In Boito risulta esclusivo l'esito per la variante letteraria con sonorizzazione della consonante. Nell'Ottocento, spalto è variante più regolare ormai, mentre spaldo è relegato alle opere poetiche di Emilio Praga e Carducci (cfr. GDLI; BI). Boito se ne serve anche nei libretti Amleto (Nardi 1942: 502) ed Otello (Nardi 1942: 879, 880, 882, 894), probabilmente attingendo dalla Commedia dantesca (Inf. IX, 133).

${ }^{37}$ Variante arcaica per il più comune scuriata (cfr. GDLI), è attestata in Dante (Inf. XVIII, 65).

${ }^{38} \mathrm{Nel}$ solo Trapezio, novella dal tono letterario. Nel precedente Pugno chiuso, infatti, comparivano tre usi di unghie [PC: 231, due casi in 233].

39 Sebbene capegli non sia considerato poetico o particolarmente marcato nel corso del XIX secolo (cfr. TB), tale forma nel primo Ottocento viene ormai sostituita da capelli 
Si contano inoltre, sia nei verbi che nei sostantivi, forme consonantiche geminate (innondare [IB: 187], arruffatto/- $i$ [PC: 224; MP: 202], scattola [PC: 229], caratti [PC: 229, 232], tranguggiarlo [MP: 206], obbliare [TR: 259] ed obblio [TR: due casi in 259]) o scempiate (inaffiare [MP: 206], scalfitura [TR: 259], sopranome [TR: 273] $]^{40}$ ), alcune desuete o rare già per il XIX secolo ${ }^{41}$. Altri scempiamenti, invece, sono legati alla resa etimologica: susurrò [IB: 193], imagine [IB: 187; PC: 235; TR: 244], giacio [TR: 240$]^{42}$.

\section{ELISIONE}

L'elisione sottende una tensione dell'autore verso l'esito eufonico dell'espressione e coinvolge regolarmente gli articoli determinativi singolari, sempre elisi davanti a vocale (si conta anche un caso di plurale ne «Gl'idropici» [PC: 244]) $)^{43}$, meno perentoriamente gli indeterminativi (tra le eccezioni, «una espressione» [MP: 212], «una idea» [TR: 243]); è più consistente nelle preposizioni (sempre elise davanti a vocale, sia di articolo indeterminativo che di sostantivo, pronome o verbo, salvo che in alcuni rari casi, per esempio «di un nativo» [AN: 167], «prima di incominciare» [MP: 206]), nei pronomi clitici ${ }^{44}$, nei pronomi atoni lo e la; in certe occasioni, investe anche aggettivi (ad esempio «prim'urto» [IB: 189], «una sol'ape» [IB: 185], od i cardinali («sett'anni» [IB: 183; MP: 202]; PC: «trent'ore» [PC: due casi in 227]) e le congiunzioni («mentr'essa/ei/egli» [IB: 187, due in 196; MP: 203, 213]).

(Migliorini 1960: 627). Boito se ne serve solo nella novella Iberia, dal tono poetico, mentre nelle altre, ad esempio nella Musica in piazza [MP: 206], ricorre all'uscita in alveolare - $l$-.

${ }^{40}$ Ormai soppiantato nell'Ottocento da soprannome, ma attestato in Dante (Purg. XVI, 139; Par. XV, 138).

${ }^{41} \mathrm{Cfr}$. TB; BI; GDLI. Si tenga presente che alcune voci oltre a quelle già contemplate, come arrottondare [TR: 270] o tattuaggio [TR: 248] (oscillante con tatuaggio [TR: 260]), o lo scempiato patuita [TR: 242], potrebbero considerarsi refusi del tipografo.

${ }^{42}$ Non attestato nel GDLI, ma registrato nella BI solo fino a Pier Francesco Giambullari (sedicesimo secolo), in Boito giacio non consiste in un'incertezza d'uso, in quanto si ripete, nella librettistica, anche nella Falce (Nardi 1942: 566) e nell'Otello (Nardi 1942: 896, 954).

${ }^{43}$ Unica eccezione: «la instabilità del fanciullo» [TR: 246].

${ }^{44}$ L'elisione davanti a vocale s' incrementa di novella in novella, confermandosi come un carattere proprio della scrittura boitiana. Nel Trapezio, per esempio, i clitici risultano sempre elisi tranne che in sporadiche eccezioni [TR: 272, 239, 265, passim.]. 


\section{MORFOLOGIA}

In ambito morfologico, si sottolinea la sistematica predilezione di Boito per le forme sintetiche delle preposizioni $d i, a, d a$, in, su, per + articolo determinativo: «pel crescente ingrossar» [AN: 173], «per noi e pei nostri figliuoli» [IB: 183], «misurò collo sguardo» [IB: 186], «cinguettante co' passeri, librantesi colle rondini» [MP: 199], ecc. Relativamente al solo per, le preposizioni femminili vengono sempre risolte nelle analitiche $p e r+l a$ e per + le, evitandone un esito più marcato.

Per quanto riguarda il pronome, Boito si serve esclusivamente di egli, accogliendo quel «tradizionale primato letterario» che si sarebbe mantenuto in alcuni scrittori ancora fino al secondo Ottocento ${ }^{45}$. Per il femminile, è assente ella a favore di essa. Egli subisce alcune oscillazioni in Iberia e nel Trapezio con la forma arcaica e letteraria ei («ei si direbbe» [IB: 182], «ei la guardò» [IB: 196], «ei non sapeva contenere» [TR: 248]), contribuendo alla cifra poetica delle due novelle. È interessante notare come i pronomi tonici (meco [TR: 250], teco [TR: due casi in 240], seco [TR: 243]), ormai sempre più relegati nel secondo Ottocento al genere poetico ${ }^{46}$, si attestino soltanto nel Trapezio, l'ultima novella e dal più spiccato carattere letterario.

A proposito delle flessioni verbali, si contano attestazioni più o meno marcate del bisillabo ponno [IB: 181], di scande [Ibid.] per 'scandisce', di appariscelappariscono [PC: 223, 236; MP: 218] $]^{47}$, di alcune uscite in $-a$ della prima persona nell'indicativo presente («io era» [TR: 241], «rimaneva io» [TR: 259]) o nell'indicativo trapassato prossimo («[io] non aveva inteso mai» [TR: 247], «[io] non aveva veduto» [Ibid.]), di casi di caduta della fricativa sonora intervocalica («e avea steso il pugno» [IB: 191], «l'avea biondo» [PC: 224], «che mi facea traballare» [TR: 270]), di alcune occorrenze tronche per la terza persona singolare del passato remoto («li vendé») [AN: 168], «diè un grido» [IB: 196], «Vendé i suoi stracci» [PC: 227]). Si tratta nel complesso di forme che vanno sempre più relegandosi, nel secondo Ottocento, al genere poetico; ed anche in questo, sono impiegate sempre meno ed acquistano un tono letterario ${ }^{48}$. Si attestano inoltre due casi marcatamente arcaici in «erano iti al lavoro» [MP: 215] e nel verbo guatare [TR: 246], quest'ultimo utilizzato da Boito ripetutamente anche nei libretti (Nardi 1942: 121, 530, 576, 587, 616) ${ }^{49}$.

${ }^{45}$ Cfr. Serianni (2013: 203, n. 3).

${ }^{46} \mathrm{Cfr}$. BI.

${ }^{47}$ Appajono [AN: 167] è attestato una sola volta nell' Alfier nero.

${ }^{48}$ Cfr. BI; GDLI; Koban (2017: 142, 144).

49 Si tratta del Mefistofele, dell'Amleto, dell'Ero e Leandro, della Gioconda. Negli ultimi Otello, Falstaff e Nerone, guatare non è più attestato. 


\section{LESSICO}

Sul piano lessicale, componente essenziale di letterarietà è il ricorrere di voci schiettamente poetiche, alcune già desuete per il suo tempo, che Boito ricavava dalla lettura dei testi letterari e soprattutto dalla Commedia dantesca $^{50}$. Ma a fianco di espressioni tradizionali e poetiche, sia anticheggianti sia ancora attive nell'Ottocento, s'impongono novità linguistiche desunte dalle varie discipline e dall'introduzione costante di forestierismi. Nel complesso, la selezione eufonica di un termine contribuisce al carattere letterario già individuato nelle novelle $\mathrm{e}^{51} \mathrm{ed}$ all'affermazione di una scansione alle volte euritmica del discorso ${ }^{52}$, dove il materiale linguistico si rivitalizza tanto da liberare i termini più desueti della propria patina di arcaicità ${ }^{53}$. Tra le numerose occorrenze, si segnalano: voci rare o letterarie (alipede [MP211], corsiero [TR: 257, due casi in 258, 259], cuna [IB: 194] ${ }^{54}$, graveolente [MP: 199; TR: 262], romitaggio [IB: 185]), talvolta antiche o già in fase

${ }^{50}$ L'importanza della Commedia, assunta come modello per una ricerca di stile "ben oltre le antologizzazioni di gusto romantico" (Paolazzi 1974: 292), è riscontrabile lungo tutta la parabola creativa di Boito, coinvolgendo i versi, i libretti e le novelle, dove è possibile imbattersi in continui calchi o prestiti danteschi. L'autorità che Boito assegna a Dante è attestata nel Trattenimento musicale da Giovanni Noseda, pubblicato nel "Figaro" il 1864: "Quando si pensa che in materia di lettere si suol dire anche oggi, e da persone accostumate, la frase $i$ quattro classici! e c'è il costume, o lo scostume, di legare in marocchino rosso Dante con Petrarca e Ariosto e Tasso, senza segnare, tra il primo che è Dio e gli altri tre che son tutti uomini, una linea di separazione religiosa, si riesce a capire ogni sorta di accoppiamenti e di confusioni deformi. E Beethoven è Dio in musica come Dante in poesia" (Nardi 1942: 1110). Elementi danteschi sono riscontrabili ad esempio nel lessico dell'Otello per Verdi (cfr. Pozzo 1983: 139-140). Si rimanda alle parole rivolte a Boito da Bernardino Zendrini, che rilevava nella prosa “il ricorrere, forse un po' troppo frequente, di voci antiquate - segnatamente dantesche - che offendono, qua e là, l'orecchio" (cfr. Nardi 1924: 172).

51 "Sia a livello di contenuti sia a livello di strutture, l'operazione di Boito si orienta secondo un atteggiamento agonistico rispetto alla tradizione: le fonti sono eterogenee, straniere e italiane (e talvolta lontane, come Dante): i materiali del passato prossimo sono travisati e forzati, quelli del passato più antico sono riutilizzati in funzione polemica" (Spera 1994: 5). Come nota anche Di Benedetto a proposito di Case nuove (nel Libro dei versi), "la prosaicità è ricercata anche mediante le scelte lessicali basse, deliberatamente messe a contrasto con quelle auliche" (Benedetto 1994: 16); ciò risulta evidente dallo spoglio lessicale della Musica in piazza.

${ }^{52}$ Cfr. Benedetto (1994: 16); Nardi (1924: 172); Spera (1994: 5).

${ }^{53}$ Così Croce a proposito di Dualismo (cfr. Croce 1943: 259).

${ }^{54} \mathrm{Si}$ tenga presente però che cuna è contemplata anche come milanesismo del primo Ottocento (cfr. Angiolini 1897; Zangrandi 2002: 202), ed anche voce veneta (cfr. Prati 1968). Non si dimentichi che Boito era nato a Padova. 
di calo nel secondo Ottocento (ammansare [MP: 200, 208], aromato per 'aroma' [TR: 256], avvoltava [TR: 257], entomati [AN: 175] ${ }^{55}$, epiderme [Ibid.], solfa per 'solfeggio' [MP: 219], squarrato per 'rauco, gutturale' [MP: 199]); dantismi (artimone [TR: 245] ${ }^{56}$, baldezza [TR: 257], valchi per "passi compiuti camminando o correndo' ${ }^{57}$ [MP: 210; TR: 257]); voci marcate da una grafia preziosa (catalepsi [TR: 260, 263], coleöptero [MP: $202]^{58}$ ); latinismi (cerebro [TR: 259] ${ }^{59}$, diafragma [MP: 204], musculatura [MP: 205], vespero [TR: 213]); voci di attestazione recente nel panorama ottocentesco (avana per 'sigaro confezionato con tabacco dell' Avana' [AN: $170]^{60}$, avatare [MP: 206] ${ }^{61}$, velocipedista [MP: due casi in 211]); espressioni tecniche, in prevalenza dal lessico medico-scientifico (ammansamento [MP: 210], cronico [PC: 224], vibrione [TR: 246], zonàrio [Ibid.]), ma anche musicale (crescendo [MP: 204], il sostantivo strappate [MP: due casi in 108, 212], pandura [MP: 212], mandòla [Ibid.]) ed artistico (drappeggiamento [IB: 191; TR: 262], ornamentista [MP: 202], sconnessure [MP: 217]); voluti dialettalismi, come i milanesi mo [MP: 217] ${ }^{62}$ e scior [MP: 215] ${ }^{63}$; forestierismi ${ }^{64}$ (dal francese, pince-nez [AN: 167], bosses [Ibid.], boxeurs [AN: 172], truands [PC: 223], bambù [TR: 241]; dall'inglese, grooms [AN: due casi in 168], tunnel [MP: 200], clown [TR: 254, 257, ecc.]; dal cinese sam-pan per un tipo di imbarcazione [TR: 243], koo per «mercante» [TR:

${ }^{55}$ Forse ricavato dall'entomata dantesco (cfr. Purg. X, 128).

${ }^{56}$ Anche nella Gioconda (cfr. Nardi 1942: 643), nell'Otello (cfr. Nardi 1942: 881), nel Nerone (cfr. Nardi 1942: 226).

${ }^{57}$ Cfr. Purg. XXIV, 97.

${ }^{58}$ Forse un calco dal latino scientifico coleoptera (cfr. GDLI).

${ }^{59}$ Attestato anche in Re Orso (cfr. Villa 2009: 304), Otello (cfr. Nardi 1942: 891), Falstaff (cfr. Nardi 1942: 975).

${ }^{60}$ Cfr. GDLI.

${ }^{61}$ Dal francese avatar; l'epitesi in -e non è contemplata nel GDLI né in altro luogo.

${ }^{62}$ Cfr. Arrighi (1896). Ma è anche contemplato come esclamazione toscana (cfr. Fanfani 1976).

${ }^{63}$ Boito fornisce subito tra parentesi una traduzione, «(signore)» [MP: 215], e scior è sottolineato in corsivo dallo stesso autore, che preferisce quindi definire l'esclamazione come tratto marcato nel testo. Altri dialettismi sono i versi di Barbapedàna [MP: 205], non tradotti, e l'espressione «c'è sotto cantina!» [MP: 203], definito dallo stesso Boito «espressivo idiotismo lombardo» [Ibid.] (cfr. Danzi 2001: 252; Villa 2009, 582). Ma questi milanesismi sono piuttosto degli unica nella novellistica boitiana, relegati alla Musica in piazza, in quanto, anche per cause di diversa ambientazione, non ricompaiono in nessun'altra delle restanti novelle.

${ }^{64}$ I quali, come i milanesismi, sono spesso giustificati dall'ambientazione delle novelle in cui sono attestati. 
242]; dallo spagnolo hidalgo [IB: 181], lo Xeres per 'sherry' [AN: 169] ${ }^{65}$; dal polacco ${ }^{66}$, come briska per 'calesse' [PC: 223, 232, 234] o la scrittura kopiec per 'copeco' [PC: due casi in 224, due in 225, 234]). Interessante il caso di caleide [IB: 192], apparsa anche nei componimenti Un torso (Villa 2009: 64) e Poiché ho l'anima cupa e sbigottita (Villa 2009: 81), ambedue raccolti nel Libro dei versi. La voce, non contemplata in altri autori al di fuori di Boito, ad eccezione del solo D'Annunzio (cfr. D'Annunzio 1982: 38), sembrerebbe un'invenzione boitiana per denotare un tipo di insetto ${ }^{67}$.

\section{SINTASSI}

Per concludere, la sintassi paratattica si dimostra estremamente ordinata: l'utilizzo del che polivalente (alla ribalta nell'àmbito della letteratura verista $\left.^{68}\right)$ risulta pressoché irrilevante, relegabile ad esempi di valore temporale e sempre calati in contesti dialogici. Sono presenti inoltre dislocazioni dei termini (posposizioni dell'aggettivo possessivo rispetto al nome, come in «Quest'aurora è il tramonto mio» [IB: 184] e «stava sepolto [...] il debitore suo» [PC: 228], o viceversa anteposizioni dell'aggettivo qualificativo, come in «liturgico salmo» [IB: 191] o "sua eletta ed ironica natura» [MP: 216]; aggettivi nella disposizione di coppie a occhiale, ad esempio «sulla sacrata croce vera» [IB: 192]); figure di accumulazione come l'anafora («La musica in piazza è un umile concetto, un tema innocente. La musica in piazza è la musica in libertà» [MP: 199], «Sorridi pure senza temere d'offendermi [...]. Sorridi pure delle contraddizioni umane» [TR: 240]), l'anadiplosi («Bisognava salvarlo! salvarlo con tutta la forza possibile» [AN: 177]), o costruzioni seriali quali l'accumulo di elementi nominali («Sotto quella tenebrosa coperta si possono supporre tutte le schiatte umane di tutti i tempi; lo spagnuolo, il saraceno, l'hidalgo, l'inquisitore, la corazza di ferro del quattrocento, il giustacuore di cuojo del cinquecento, la giubba di velluto del seicento vi si potrebbe parimente celare [IB: 181]») e vari sintagmi preposizionali, che dettano un affastellamento straripante di parallelismi come dittologie («è caritatevole, magnanimo; i suoi contadini lo venerano, lo benedicono» [AN: 168]) e tricola («m'esercitavo al bersaglio colle pietre, colle freccie, colle frombole» [TR: 242]); presenze di antitesi («Attacco e difesa, azione, reazione, angolo percosso, angolo riflesso» [TR: 245]) e chiasmi («fra il caldo vapore dei profumi sacri» [PC: 224]). Si tratta, nel complesso, di procedimenti che incrementano la resa letteraria del tessuto

\footnotetext{
${ }^{65}$ Ricorrerà anche in Falstaff (Nardi 1942: 958). Cfr. Zanon (2007: 963, n. 28).

${ }^{66} \mathrm{Si}$ ricordi che Boito era figlio di madre polacca (cfr. Nardi 1942a: 91-116).

${ }^{67}$ Per il significato del termine, si rinvia al saggio di Crotti (1991).

${ }^{68}$ Cfr. Colombo (2011: 106, 109, 118).
} 
sintattico. Oltre a quanto, la voce del narratore si percepisce attraverso la reiterazione in più luoghi dell'avverbio come, il quale, introducendo l'elemento comparante, "rivela il narratore come autore anche dell'ornatus che si coniuga al racconto" (Zangrandi 2002: 134) ${ }^{69}$ : «Appena cessò il nostro riso, s'udì ancora come un eco gutturale e sardonico il riso dei tacchini. [...] Ogni nostra risata eccitava una risata dei polli e viceversa, e sempre più forte, come un crescendo di Generali» [MP: 203].

\section{CONCLUSIONE}

Pur rilevando dei caratteri distintivi tra le cinque novelle, si può osservare come nel lessico variegato ogni scelta tenda a convergere in una letterarietà talvolta preziosa, come in Iberia o nel Trapezio, talvolta cólta, come nella Musica in piazza. Il mantenimento di un vocabolario apertamente letterario non deve intendersi come ossequio o retaggio inerziali rispetto alla tradizione, bensì come ricerca dell'espressione volta all'organizzazione del discorso mediante una scrittura eufonica ed euritmica. Se, da una parte, il percorso poetico e musicale di Boito ha i presupposti nel movimento scapigliato degli anni Sessanta, con cui condivide alcuni ideali estetici atti a competere con la consolidata tradizione letteraria e in generale $\operatorname{artistica}^{70}$, dall'altra sembrerebbe oltrepassarlo - e non soltanto per motivi cronologici - in quanto i suoi lavori poetici (Il libro dei versi e Re Orso) e novellistici (situati tra il '67 e il '74) denotano una ricerca formale ben al di là della prassi scapigliata. Coerentemente con gli scrittori di questa temperie, egli si servì sia in poesia che in prosa di un lessico aggiornato, attento ad una terminologia tecnica e scientifica, anche dialettale, giungendo inoltre - secondo la sua cifra poetica personale - ad attribuire alla parola un ruolo fondamentale non solo per il significato che essa racchiude, ma anche (e soprattutto) per la rilevanza fonica che apporta all'espressione attraverso il recupero di sonorità spesso arcaizzanti (ricavate in gran parte dalla Commedia) che non precludono minimamente l'occorrenza di termini tecnici inusuali in contesto letterario, o di forestierismi: questa pratica contribuisce ad aggiornare il materiale desueto, aggiustandolo entro forme espressive moderne e tendenti al tono medio, lontano ormai dall'aulicità risorgimentale appena conclusa, ed organizzandolo entro una sintassi piana, non complicata da subordinazioni, dove i termini si susseguono per aggiunzione. Se la tendenza ad un registro medio era un carattere condiviso dagli scrittori

${ }^{69}$ La frequenza delle comparative sarà inoltre una cifra della paratassi dannunziana (cfr. Beccaria 1975: 293).

${ }^{70} \mathrm{Si}$ pensi alle battaglie musicali per il rinnovamento della forma nell'opera propugnato da Boito (cfr. Nardi 1942: 1080-1082). 
scapigliati (Romanini 2014), il perpetuare di forme retoriche ed altri tratti schiettamente letterari, che denotano il rifiuto dell'autore di liberarsi di un vocabolario prezioso e di un'architettura complessivamente euritmica, fa della scrittura boitiana un preludio alle prose artistiche di D'Annunzio, dove del resto si rinvengono effetti di parallelismo ed accumulo, un insistente uso di dicola e tricola, l'impiego costante del comparante come e, anche qui, una ricerca del vocabolo preciso e capace di dettare impressioni eufoniche $^{71}$, talvolta marcandole attraverso l'utilizzo degli accenti ${ }^{72}$. È però presente un tratto demarcativo tra i due autori: in Boito il ruolo della parola non si abbandona mai a un'assoluta evocazione musicale, dove il significante sia scelta principale nella selezione del lessico. Infatti, il dettato poetico boitiano si mantiene costantemente in equilibrio tra valore musicale ed esigenze funzionali connesse all'economia della novella. Ad ogni modo, nel caso di Iberia e del Trapezio si può tranquillamente affermare la loro appartenenza alla categoria di una prosa poetica, ponderata e raffinata, aristocratica, certamente senza eccessi, ma dotata di fenomeni inerenti alle apocopi vocaliche ed altri vari nessi vocalici e consonantici che sembrano preludere ai numerosi stilemi che saranno propri, a breve, delle scritture di Carducci $^{73}$ e, soprattutto, di D'Annunzio ${ }^{74}$. Siamo tra gli anni Sessanta e Settanta dell'Ottocento: è questo il decennio in cui affiorano i cosiddetti "scrittori d'arte" 75 e, sotto certi aspetti, Arrigo Boito, con le sue novelle, sembra anticiparne, secondo la sua natura poetica, alcuni caratteri.

\section{BIBLIOGRAFIA}

\section{Sigle delle novelle}

$\mathrm{AN}=$ L'alfier nero.

$\mathrm{IB}=$ Iberia .

$\mathrm{MP}=$ La musica in piazza.

$\mathrm{PC}=$ Il pugno chiuso.

$\mathrm{TR}=$ Il trapezio.

I testi di tutte le cinque novelle sono contenuti in Villa (2009).

${ }^{71}$ Cfr. Beccaria (1975: 300); Colombo (2011: 133). Per i rapporti tra Boito e D'Annunzio, cfr. Nardi (1942a: 438-439).

${ }^{72}$ Cfr. Serianni (2013: 198).

${ }^{73}$ Cfr. Migliorini (1960: 679).

${ }^{74}$ Cfr. Serianni (2013: 194-200).

${ }^{75}$ Cfr. Migliorini (1960: 679). 
Testi d'autore

Boito, A. (1867). L'alfier nero. Il Politecnico-Repertorio di studj letterarj, scientifici e tecnici, XXX Serie quarta, Parte letterario-scientifica, Vol. terzo, 269-282.

Croce, B. (1943). Letteratura della nuova Italia. Saggi critici (Vol. 1). Bari: Laterza.

D’Annunzio, G. (1982). Versi d'amore e di gloria (Vol. 1). Milano: Mondadori. Motta, A. (2015). Scritti politici e d'attualità. Venezia: Marsilio.

Nardi, P. (a cura di). (1942). Arrigo Boito. Tutti gli scritti. Milano: Mondadori.

Villa, A. I. (a cura di). (2009). A. Boito. Opere letterarie. Milano: Edizioni Otto/Novecento.

Zangrandi, A. (2008). Nota al testo. In P. V. Mengaldo \& A. Zangrandi (a cura di), Ippolito Nievo. Angelo di bontà. Storia del secolo passato (pp. 21-35). Venezia: Marsilio.

\section{Studi critici}

Beccaria, G. L. (1975). L'autonomia del significante. Figure del ritmo e della sintassi: Dante, Pascoli, D'Annunzio. Torino: Einaudi.

Benedetto, A. D. (1994). «Case nuove» o le rovine di Milano. In G. Morelli (a cura di), Arrigo Boito. Atti del convegno internazionale di studi dedicato al centocinquantesimo della nascita di Arrigo Boito (pp. 15-33). Firenze: Olschki.

Buroni, E. (2013). Arrigo Boito librettista, tra poesia e musica. La «forma ideal, purissima» del melodramma italiano. Firenze: Franco Cesati Editore.

Colombo, M. (2011). Il romanzo dell'Ottocento. Bologna: Il Mulino.

Coletti, V. (1993). Storia dell'italiano letterario. Dalle origini al Novecento. Torino: Einaudi.

Crotti, I. (1991). Ancora a proposito delle caleidi (tra Arrigo Boito e D'Annunzio). In G. Borghello, M. Cortelazzo \& G. Padoan (a cura di), Saggi di linguistica e di letteratura in memoria di Paolo Zolli (pp. 541-547). Padova: Editrice Antenore.

Danzi, L. (2001). Lingua nazionale e lessicografia milanese. Manzoni e Cherubini. Alessandria: Edizioni dell'Orso.

Koban, F. I. (2017). Cavour e l'italiano. Analisi linguistica dell'epistolario. Pisa: Edizioni ETS.

Marazzini, C. (1994). La lingua italiana. Profilo storico. Bologna: Il Mulino. Migliorini, B. (1960). Storia della lingua italiana. Firenze: Sansoni.

Nardi, P. (1924). Scapigliatura. Da Giuseppe Rovani a Carlo Dossi. Bologna: Zanichelli. 
Nardi, P. (1942a). Vita di Arrigo Boito. Milano: Mondadori.

Paolazzi, C. (1974). Cultura e «paradiso perduto»: note di fortuna dantesca tra gli scapigliati. In F. Mattesini (a cura di), Novità e tradizione nel secondo Ottocento italiano (pp. 262-337). Milano: Vita e Pensiero.

Pozzo, G. D. (1983). “Otello" tra Verdi e Boito. Belfagor, 38, 129-154.

Romanini, F. (2014). Forme brevi della prosa letteraria. In G. Antonelli, M.

Motolese \& L. Tomasin (a cura di), Storia dell'italiano scritto (Vol. 2, pp. 203-254). Roma: Carrocci.

Serianni, L. (2013). Storia dell'italiano nell'Ottocento. Bologna: Il Mulino. Spera, F. (1994). Le sperimentazioni poetiche di Arrigo Boito. In G. Morelli (a cura di), Arrigo Boito. Atti del convegno internazionale di studi dedicato al centocinquantesimo della nascita di Arrigo Boito (pp. 1-13). Firenze: Olschki.

Zangrandi, A. (2002). Lingua e racconto nel romanzo storico italiano (1827-1838). Padova: Esedra.

Zanon, T. (2007). Il 'bianco' e il 'nero'. Boito e il libretto per l'«Otello» di Verdi. In allievi padovani (a cura di), Studi in onore di Pier Vincenzo Mengaldo per $i$ suoi settant'anni (Vol. 1, pp. 953-965). Firenze: SISMEL-Edizioni del Galluzzo.

\section{Strumenti}

Angiolini, F. (a cura di). (1897). Vocabolario milanese-italiano. Torino: Paravia.

Arrighi, C. (a cura di). (1896). Dizionario milanese-italiano. Milano: Hoepli.

$\mathrm{BI}=$ Biblioteca Italiana, disponibile al sito http://www.bibliotecaitaliana.it/ consultato nel mese di settembre 2019.

Fanfani, P. (a cura di). (1976). Vocabolario dell'uso toscano. Firenze: Le Lettere.

GDLI = Grande Dizionario della Lingua Italiana . (1973) Torino: Utet.

Prati, A. (1968). Etimologie venete. Venezia-Roma: Istituto per la collaborazione culturale.

$\mathrm{TB}=\mathrm{N}$. Tommaseo \& B. Bellini (a cura di). Dizionario della lingua italiana . CD-Rom (2018). Bologna: Zanichelli. 


\section{OBSERVATIONS ON THE LANGUAGE OF ARRIGO BOITO'S NOVELLAS}

\section{Summary}

The article examines phonological, morphological, graphic, lexical and syntactic aspects of the five Arrigo Boito's novellas (L'alfier nero, Iberia, La musica in piazza, Il pugno chiuso and Il trapezio), which were written in the short time between 1867 and 1874. Among linguistic phenomena in Boitian prose, the most significant features which contribute to a generic eurhythmic treatment of the text have been collected and contemplated, such as recurrence of poetisms and a decidedly outmoded or technical vocabulary, Dantean expressions, dialectalisms or foreignisms: through an overall examination, the experimental trait of Boito's writing was noted and it was possible to compare the individual expression of the writer and musician with the stylistic features of 19th-century Italian language (especially with Scapigliatura movement), in relation to the other Boito's poetical works and his opera librettos.

Keywords: Arrigo Boito, novellas, language, Nineteenth century. 